\title{
Characterization of a Carotid Distension Waveform from Audio Signal Acquired with a Stethoscope
}

\author{
Iván Maldonado, Alfredo Illanes, Axel Boese, Michael Friebe \\ Otto-von-Guericke-Universität Magdeburg, Germany
}

\begin{abstract}
Non-invasive methodologies for diagnostic and prevention of cardiovascular problems has a special interest in the measurement of blood pressure because of its implications in arterial stiffness, mostly as a consequence of arteriosclerosis. Assuming an established relationship in the literature between blood pressure and the carotid artery flow measured with Doppler ultrasound, in this work a carotid distension waveform has been extracted from carotid audio signal obtained with a conventional stethoscope. For evaluation of the proposed approach fiducial points from the obtained carotid audio signal has been compared with fiducial point in the Doppler blood flow velocity waveform. Preliminary results show a good correspondence between the processed audio signals and Doppler spectrum.
\end{abstract}

\section{Introduction}

Since the late 40's with the development and implementation of ultrasound (US) in medicine, the progress of noninvasive diagnostic image devices and procedures has been motivated by the reduction of pain, trauma, recovery time and cost, resulting from diagnostic and preoperative surgical interventions. In addition, medical image devices have allowed the early detection of multiple diseases because of its association to cardiovascular and cerebrovascular diseases [1-3]. A good example of that is carotid stenosis assessment, where US has played an important role for its early detection, diagnostic and quantification [4].

Regardless all advantages provided by non-invasive diagnostic image procedures and advances in image processing, alternative solutions with a lower cost and accessible to a larger population have been well accepted in the last decade. Nevertheless, their accuracy and systematic differences in numerical results need to be improved.

The implementation of Micro Electro Mechanical Systems (MEMS) in the medical field is emerging as a new alternative for non-invasive diagnostic procedure thanks to the association and surrogate of different physical measurements as pulse pressure, which is a parameter of high risk factor in cardiovascular complications $[5,6]$.

An original approach to estimate carotid pulse pressure implementing MEMS accelerometer has been presented in [7]. This work can be extended to audio because of its intrinsic relationship with the acceleration of blood flow [8] for the detection of heart diseases [9].

In this work it is shown that from carotid audio signals obtained with a standard stethoscope a waveform with similar characteristics than the carotid distension pressure waveform can be obtained. The consistency of the obtained waveform is confirmed by comparing fiducial points of the signal with the ones of a Doppler flow velocity waveform following a established relationship presented in $[10,11]$.

Results show that for every tested subject the fiducial points of the audio version of the carotid distension waveform fit well the ones of the Doppler signal. This opens perspectives for the integration of conventional instruments, as a stethoscope, in a digital era where less invasive and accessible devices are demanding paradigm changes in health-care systems.

\section{Materials and Methods}

In this work a signal presenting similar morphological characteristics than a carotid distension pressure waveform is extracted from audio signals. In order to show the consistence of our audio version of the distension waveform we use the established relationship presented in [10] between the distension waveform and the carotid flow velocity waveform extracted with a Doppler Ultrasound (US). This is why US Doppler signal are acquired in this work in order to compare fiducial points of the distension pressure waveform obtained from the stethoscope audio signal and fiducial points of the Doppler signal.

In this section the procedure for carotid audio data acquisition is first introduced and then the methodology for pre-processing of the audio and ultrasound signals is presented. Finally the algorithm for obtaining a carotid distension waveform version from the audio signal is explained. 


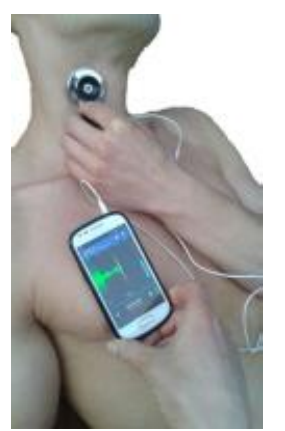

Figure 1. Acquisition of carotid audio signals using a stethoscope attached to a smartphone.

\subsection{Data Acquisition}

The acquisition of carotid audio signals and US Doppler images has been performed in the right superior carotid triangle in four volunteer subjects during 30 seconds. The audio data has been obtained with a sapling frequency of $44100 \mathrm{~Hz}$ using a conventional stethoscope of $44 \mathrm{~mm}$ diameter attached from its flexible tubing to a microphone connected to a smartphone via $3.5 \mathrm{~mm} 4$-pole (TRRS) plug (see Figure 1).

For carotid artery blood flow, 5 Doppler US images in JPG format were acquired sequentially with an ultrasound and 9L probe (GE LOGIQe, General Electric, USA) configured as Doppler Color to $5 \mathrm{MHz}$ for different scales. Each image contained between four and five cardiac cycles.

The MATLAB version R2016a has been used for the processing of US images and audio signals.

\subsection{Audio and Doppler ultrasound data pre-processing}

The diagram of Figure 2 describes the main steps for the audio and Doppler US data pre-processing in order to obtain average audio and Doppler US signals for comparison and fiducial points analysis.

The audio signals have been first decimated by 20 and then an audio sub-signal with a minimum of four complete cardiac cycles has been manually selected. Then each cardiac cycle has been segmented using the Shannon energy envelope method presented in [12]. Finally, four segmented sub-signals were aligned and averaged using the first heart sound (systolic sound S1) as the reference for alignment (see Figure 3).

For carotid flow velocity data pre-processing, first of all the US Doppler images obtained with the US device needed to be converted to a treatable signal. For that the Doppler waveform has been segmented in each one of the 5 US images and then the envelope of the Doppler waveform

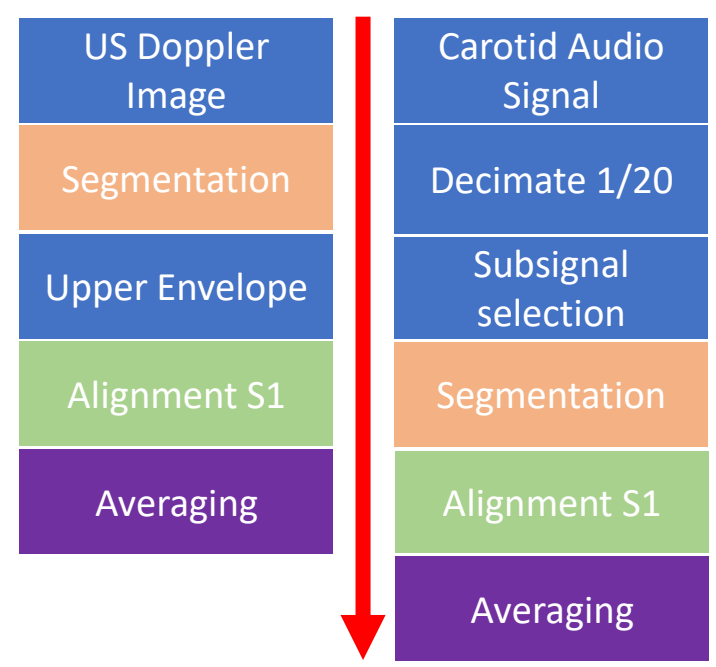

Figure 2. Block diagrams showing the main steps for US Doppler and audio data processing.

was extracted and mapped as a vector signal (see Figure 4). The resulting envelope signals were then aligned using the systolic flow velocity peak as reference for finally computing an averaged Doppler signal as shown in the last plot of Figure 4.

\subsection{Audio carotid distension waveform ex- traction}

The resulting averaged audio signal as the one shown in Figure 3C is used for computing the stethoscope audio version of the carotid distension waveform. For that the envelope of the averaged audio signal is first computed and then the resulting signal passes through a moving window integrator with a sliding window of $180 \mathrm{~ms}$ width. The resulting signal will be called Audio Carotid Distension Waveform (ACDW) in the sequel.

\section{Results}

As mentioned at the beginning of Section 2, for evaluating the validity of the obtained ACDW, fiducial points are compared between the ACDW and the measured Doppler flow velocity waveform, called DFVW in the sequel, following the work presented in [10]. In that work the carotid distension pressure waveform has been compared with the flow velocity waveform through time intervals calculated from fiducial points. In the same way our approach is evaluated buy putting in relation fiducial points of the obtained ACDW signal with fiducial points of the DFVW signal. The fiducial points used for comparison are the following (see Figure 5):

- Beginning of the waveform for both signals $A C D W_{\text {on }}$ and $D F V W_{\text {on }}$. 


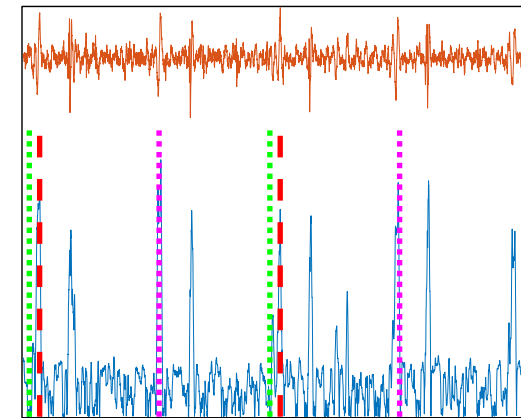

A

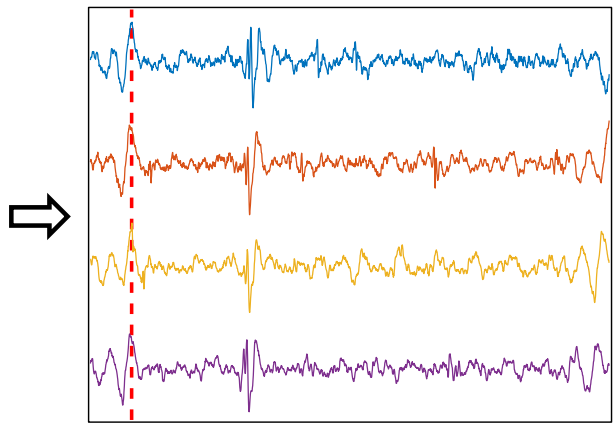

B

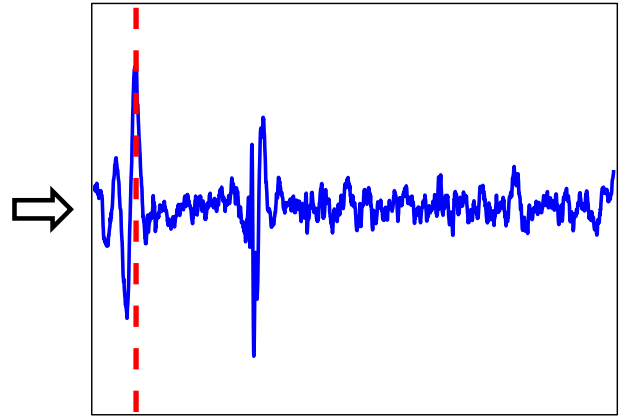

C

Figure 3. Audio Data Processing: A) Cardiac cycle segmentation (from green to magenta dotted lines) using the Shannon energy envelope and using as reference the first heart sound (red dashed line), B) Alignment of all segmented audio signals with the first heart sound (red dashed line), C) Final averaged audio signal.
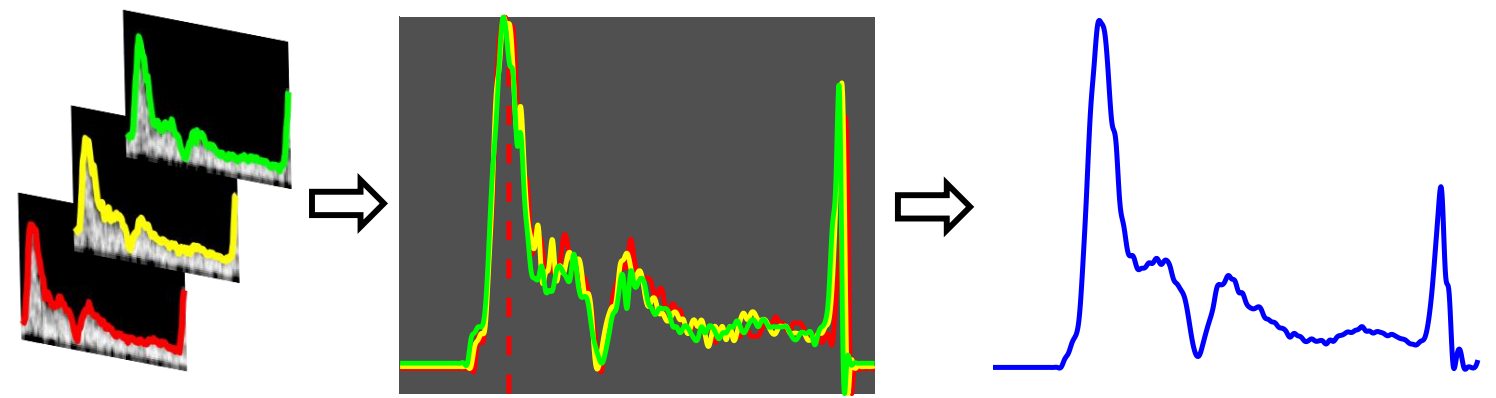

Figure 4. Ultrasound Data Processing: The blood flow signal was extracted by computing the Doppler image's tangent envelope, aligned by their peak systolic flow velocity (red dashed line) and finally averaged.

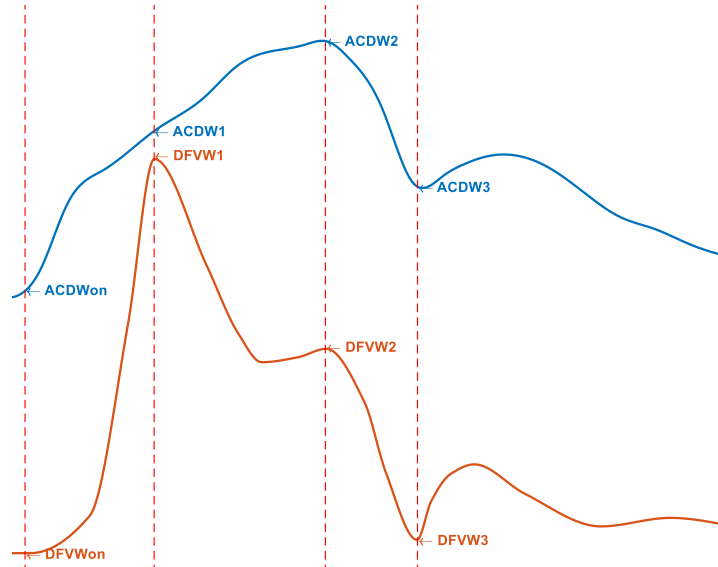

Figure 5. Illustration presenting the fiducial points used for putting in relation the audio version of the carotid distension waveform (blue) with the Doppler flow velocity waveform (red).
- The peak of the initial rise in the carotid distension $A C D W_{1}$ and the peak of the flow velocity waveform $D F V W_{1}$.

- The main peak of the carotid distension $A C D W_{2}$ and the secondary peak of the flow velocity waveform $\mathrm{DFVW}_{2}$.

- The incisura of the carotid distension and the flow velocity waveforms, $D F V W_{3}$ and $A C D W_{3}$ respectively.

Figure 6 shows the carotid distension waveform version obtained from the stethoscope audio signals ACDW for the four tested subjects. It is possible to observe that the ACDW are different morphologically from subject to subject while US doppler waveforms are quite similar. It is also evident the correct matching between the fiducial points belonging to the obtained ACDW and the ones of the DFVW signal for all the tested subjects. 

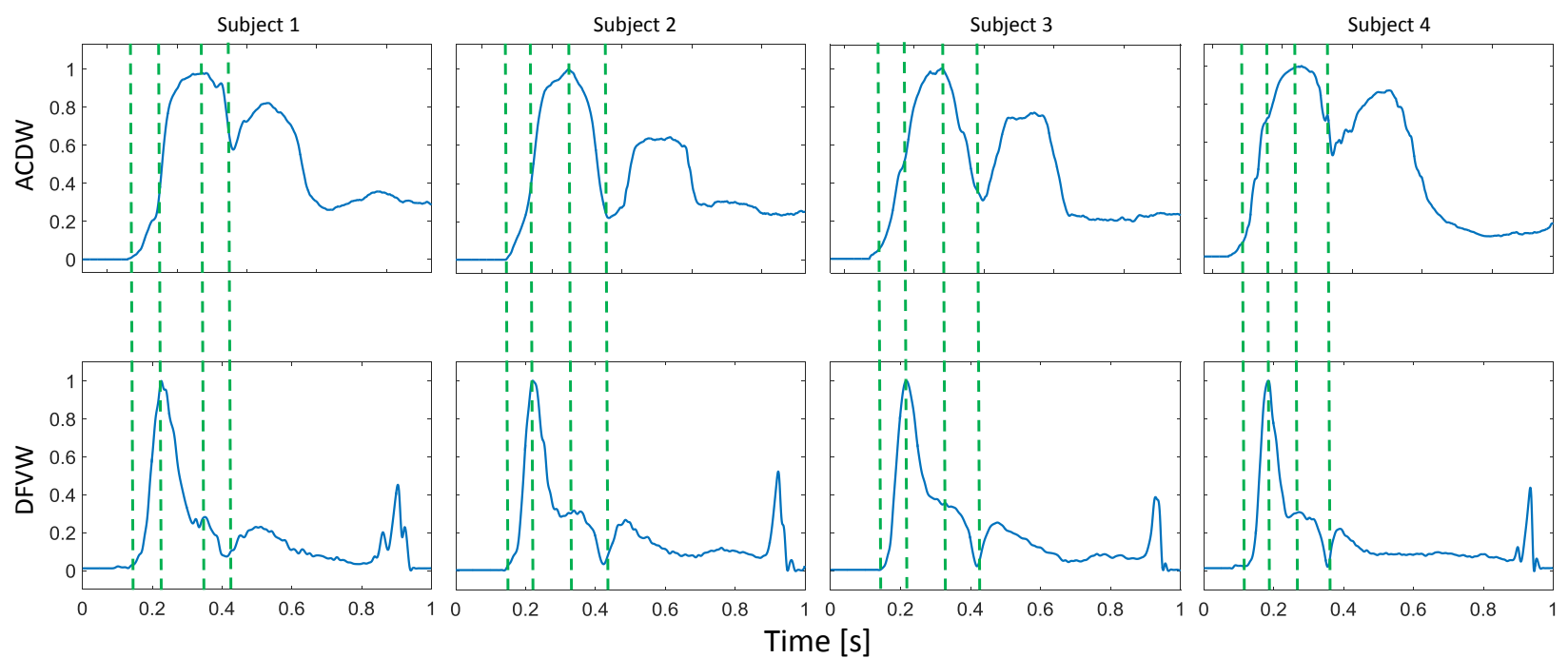

Figure 6. Resulting ACDW for the four tested subjects and fiducial points comparison with the DFVW (green dashed line).

\section{Conclusions}

In this work we have shown that a kind of carotid distension waveform can be obtained by simply recording audio from a stethoscope placed next to the carotid. This audio signal can be explained by the dilatation and contraction of blood vessels resulting in a blood pressure waveform, which confirms its similarity to the carotid distension waveform. We strongly believe that the loss of arterial elasticity could be quantified with an approach like that in the near future for preventing cardiovascular diseases.

\section{Acknowledgements}

This research was funded by the German BMBF (grant 03IPT7100X) and CONACYT Mexico.

\section{References}

[1] Slijkhuis W, Mali W, Appelman Y. A historical perspective towards a non-invasive treatment for patients with atherosclerosis. Netherlands Heart Journal 2009; 17(4):140-144.

[2] Blackshear W, Phillips D, Chikos P, Harley J, Thiele B, Strandness D. Carotid artery velocity patterns in normal and stenotic vessels. Stroke 1980;11(1):67-71.

[3] Thapar A. Carotid Ultrasound for Stroke Prediction. Ph.D. thesis, Imperial College London, 2013.

[4] Hunink M, Polak J, Barlan MM, O'leary D. Detection and quantification of carotid artery stenosis: efficacy of various doppler velocity parameters. AJR American journal of roentgenology 1993;160(3):619-625.

[5] Dart AM, Kingwell BA. Pulse pressurea review of mecha- nisms and clinical relevance. Journal of the American College of Cardiology 2001;37(4):975-984.

[6] Hermeling E, Hoeks AP, Winkens MH, Waltenberger JL, Reneman RS, Kroon AA, Reesink KD. Noninvasive assessment of arterial stiffness should discriminate between systolic and diastolic pressure ranges. Hypertension 2010; 55(1):124-130.

[7] Di Lascio N, Gemignani V, Bruno RM, Bianchini E, Stea F, Ghiadoni L, Faita F. Noninvasive assessment of carotid pulse pressure values: An accelerometric-based approach. IEEE Transactions on Biomedical Engineering 2016;63(4):869-875.

[8] Piemme TE, Barnett GO, Dexter L. Relationship of heart sounds to acceleration of blood flow. Circulation research 1966;18(3):303-315.

[9] Prakash D, Mageshwari U, Prabakaran K, Suguna A. Detection of heart diseases by mathematical artificial intelligence algorithm using phonocardiogram signals. Int J Innov Appl Stud 2013;3(1):145-150.

[10] Hirata K, Yaginuma T, ORourke MF, Kawakami M. Agerelated changes in carotid artery flow and pressure pulses. Stroke 2006;37(10):2552-2556.

[11] Kobayashi K, Akishita M, Yu W, Hashimoto M, Ohni M, Toba K. Interrelationship between non-invasive measurements of atherosclerosis: flow-mediated dilation of brachial artery, carotid intima-media thickness and pulse wave velocity. Atherosclerosis 2004;173(1):13-18.

[12] Liang H, Lukkarinen S, Hartimo I. Heart sound segmentation algorithm based on heart sound envelogram. In Computers in Cardiology 1997. IEEE, 1997; 105-108.

Address for correspondence:

Iván Maldonado (ivan.maldonado@ovgu.de)

Rötgerstraße 9, 39104 Magdeburg, Germany 